Ophthalmologe 2020 $117: 648-651$ https://doi.org/10.1007/s00347-020-01149-8 Online publiziert: 9 . Juni 2020

(c) Springer Medizin Verlag $\mathrm{GmbH}$, ein Teil von Springer Nature 2020

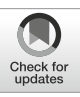

Karin U. Löffler ${ }^{1,2} \cdot$ Aja Reinhold ${ }^{3}$ Martina C. Herwig-Carl ${ }^{1,2} \cdot$ Alexandar Tzankov $^{4} \cdot$ Frank G. Holz ${ }^{1}$ Hendrik P. N. Scholl ${ }^{3}$. Peter Meyer ${ }^{3}$

${ }^{1}$ Augenklinik, Universitätsklinikum Bonn, Bonn, Deutschland

${ }^{2}$ Augenklinik, Sektion Ophthalmopathologie, Universitätsklinikum Bonn, Bonn, Deutschland

${ }^{3}$ Augenklinik, Universitätsspital Basel, Basel, Schweiz

${ }^{4}$ Institut für Pathologie, Universitätsspital Basel, Basel, Schweiz

\title{
Okuläre Post-mortem-Befunde bei an COVID-19 verstorbenen Patienten
}

PAS, Elastika-van-Gieson und MassonTrichrom gefärbt. Separat wurde auch jeweils ein Querschnitt des N. opticus untersucht.

Die histologischen Schnitte wurden von 3 Ophthalmopathologen (KUL, $\mathrm{MCH}-\mathrm{C}, \mathrm{PM}$ ) unabhängig voneinander ausgewertet.

\section{Fallbericht 1}

Ausbreitungswegen des Virus.

\section{Methodik}

Die Patienten wurden am Institut für Pathologie des Universitätsspitals Basel obduziert. Bei allen Patienten lag eine entsprechende Einverständniserklärung vor [1]. Nach Entnahme der Augen wurden diese für $72 \mathrm{~h}$ in Formalin (hygienische Vorgaben des Instituts für Pathologie/Virologie Basel) fixiert, anschließend diaphanoskopiert, makroskopisch begutachtet und bei dem ersten Patienten vertikal und den beiden anderen Patienten horizontal eröffnet. Nach Inspektion der intraokularen Strukturen wurde ein repräsentativer Block zugeschnitten und die Einbettung in Paraffin vorgenommen. Hiervon wurden jeweils Stufenschnitte angefertigt und mit Hämatoxylin-Eosin,

\section{Anmerkung der Autoren}

Einige der ansonsten recht oft zitierten Literaturstellen aus China waren leider nicht nachverfolgbar. Wir haben deswegen hier nur Arbeiten zitiert, wo zumindest ein englischsprachiges Abstract zur Verfügung stand. Nicht alle Arbeiten sind "peer-reviewed".
Die 67-jährige Patientin verstarb 14 Tage nach initialen Symptomen an respiratorischer Insuffizienz bei akuter Bronchopneumonie und interstitieller lymphozytärer Pneumonitis, bedingt durch eine SARS-CoV-2-Infektion mit bakterikung bestand eine multiple Sklerose (diagnostiziert 1991 im Alter von 39 Jahren) mit linksseitig- und beinbetonter Tetraparese und neurogener Blasenentleerungsstörung. Zum Zeitpunkt des Todes bestand seit Jahren keine immunmodulatorische Therapie mehr.

Ophthalmologisch waren eine beidseitige Optikusatrophie und eine $\mathrm{Vi}$ susminderung auf bestkorrigiert $0,3 \mathrm{am}$ rechten und 0,7 am linken Auge bekannt.

Makroskopisch zeigt sich am rechten Auge (RA) eine intrakapsuläre Pseudophakie, am linken Auge (LA) eine Katarakt. Die Papille wirkt beidseits blass. Sonst sind keine Auffälligkeiten zu erkennen.

Mikroskopisch finden sich diskrete autolytische Veränderungen, einzelne harte Drusen und eine mittelgradige eller Superinfektion. Als Grunderkran-
Optikusatrophie rechts und eine geringgradige Optikusatrophie links. Die am etwas verbreiterten - Limbus verbliebene Bindehaut ( $\bullet$ Abb. 1a) zeigt keine signifikant vermehrten Entzündungszeichen, und die verschiedenen intraokularen Gewebe sind reizfrei (• Abb. 1b).

\section{Fallbericht 2}

Der 85-jährige Patient starb 4 Tage nach Symptombeginn am respiratorischen Versagen bei COVID-19 mit einer rechtsseitigen Bronchopneumonie. Er litt zusätzlich an Diabetes mellitus Typ 2, einer myeloischen Neoplasie, einer arteriellen Hypertonie und einem schweren obstruktiven Schlafapnoesyndrom. Aufgrund der schweren Vorerkrankungen erfolgte die Therapie im palliativen Setting.

Ophthalmologisch war - bei epiretinaler Gliose und beginnender Katarakt - ein bestkorrigierter Visus von 0,7 am rechten und 0,5 am linken Auge bekannt.

Makroskopisch zeigen beide Augen eine kortikonukleäre Katarakt und in der Netzhautperipherie Pflastersteine. Am linken Auge finden sich zwischen Äquator und der Papille multiple unterschiedlich große Punkt- und Fleckblutungen. Sonst sind keine Auffälligkeiten zu erkennen.

Mikroskopisch zeigen sich neben diskreten autolytischen Veränderungen in beiden Augen altersentsprechende Befunde wie einzelne harte Drusen und die makroskopisch beobachteten Pflas- 


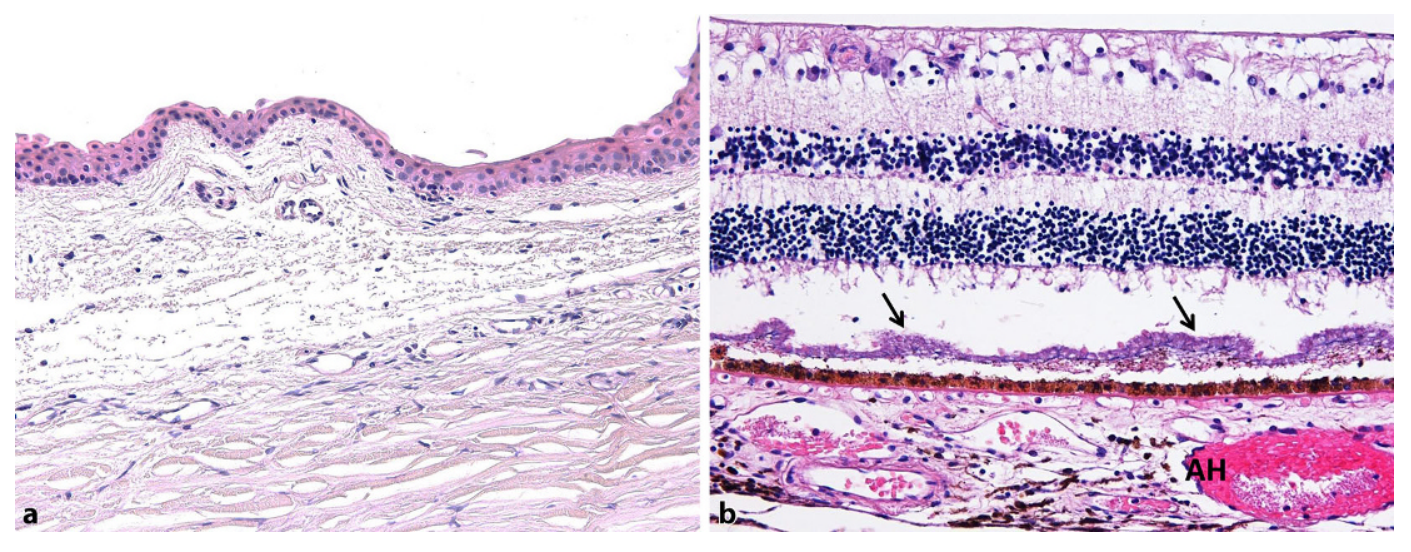

Abb. 1 \ (Patient 1, RA, Paraffinschnitt, Hämatoxylin-Eosin-Färbung, Vergr. 100!!) a Limbusnahe Bindehaut ohne Hinweis auf vermehrte entzündliche Aktivität; b mittelperiphere Netzhaut und Aderhaut ohne Hinweis auf entzündliche Aktivität (die Netzhaut ist artifiziell abgehoben; Pfeile: post-mortem-Artefakt im Bereich der Photorezeptoren mit abgescherten Außensegmenten, $A H$ Aderhaut)
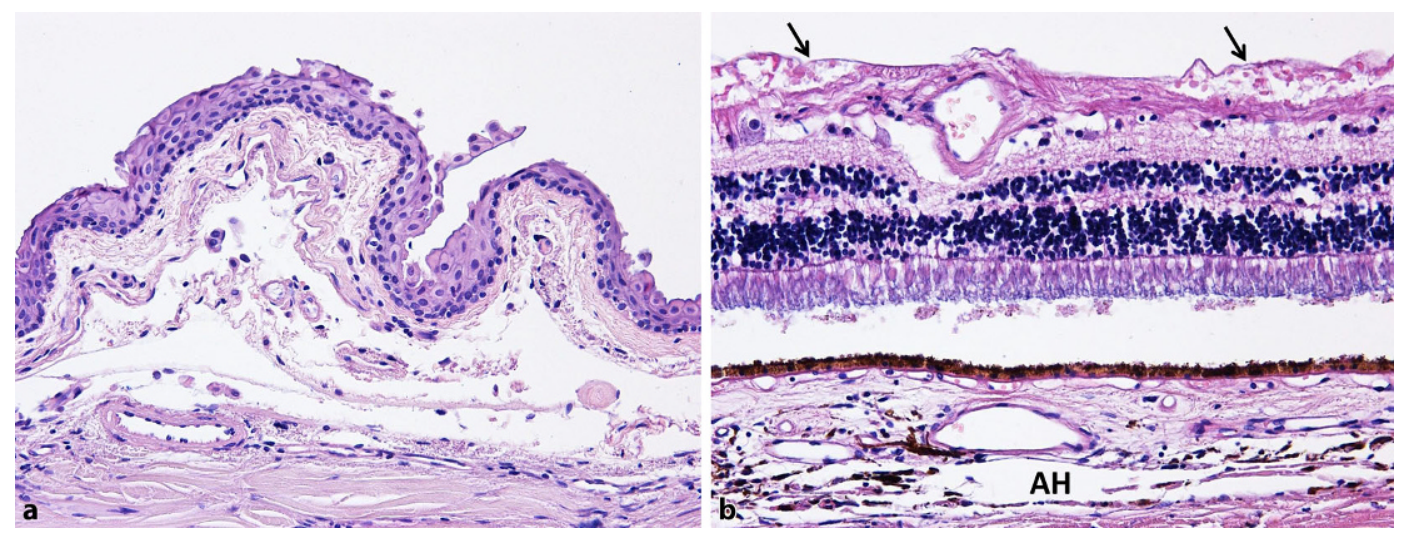

Abb. 2 \ (Patient 2, RA, Paraffinschnitt, Hämatoxylin-Eosin-Färbung, Vergr. 100:1). a Limbusnahe Bindehaut ohne Hinweis auf vermehrte entzündliche Aktivität; $\mathbf{b}$ Netzhaut und Aderhaut ohne Hinweis auf entzündliche Aktivität (Netzhaut post mortem artifiziell abgehoben, Pfeile: Erythrozyten unter der Lamina limitans interna, korrespondierend zu den retinalen Blutungen, AH Aderhaut)

tersteine. Auch der Sehnerv ist beidseits altersentsprechend. Vereinzelt sind am LA in der Nervenfaserschicht die makroskopisch auffälligen Netzhautblutungen sichtbar. Weder in der verbliebenen Bindehaut (• Abb. 2a) noch intraokular (• Abb. 2b) lassen sich entzündliche Veränderungen erkennen.

\section{Fallbericht 3}

Der 95-jährige Patient starb 4 Tage nach Symptombeginn am respiratorischen Versagen bei COVID-19. An vorbestehenden Erkrankungen waren ein Parkinson-Syndrom mit Demenz und Gleichgewichtsstörung sowie eine hypertensive Kardiomyopathie und eine kompensierte Hypothyreose bekannt.
Ophthalmologisch war eine beidseitige altersabhängige Makuladegeneration bekannt.

Makroskopisch zeigt sich beidseits ein Arcus senilis und eine ca. 3 Papillendurchmesser große gelbliche Atrophie im Makulabereich. Das RA weist eine Katarakt auf, das LA eine intrakapsuläre Pseudophakie mit deutlichem Nachstar. Sonst sind keine Auffälligkeiten zu erkennen.

Mikroskopisch finden sich diskrete autolytische Veränderungen; beidseits zeigen sich eine prominente Pinguecula und im Bereich der Makula eine ausgedehnte Atrophie der äußeren Netzhaut mit Verlust der Photorezeptoren und des retinalen Pigmentepithels und einer erheblichen Verdünnung der Aderhaut, insbesondere mit Atrophie der Cho- riokapillaris. Das RA weist eine kleine periphere oberflächliche Hornhautnarbe auf; die Linse ist artifiziell nicht vorhanden. Im LA erkennt man die Lokalisation der Hinterkammerlinse im Kapselsack, begleitet von einem ausgeprägten Nachstar im Äquatorbereich. Die am Limbus verbliebene Bindehaut zeigt geringfügige Entzündungszeichen (• Abb. 3a), die aber nicht über den im Rahmen einer intensivmedizinischen Behandlung $\mathrm{zu}$ erwartenden Befund hinausgehen. Die verschiedenen intraokularen Gewebe (-Abb. 3b) sind reizfrei.

Zusammenfassend zeigten sich bei allen Augen lediglich patientenspezifische Veränderungen entsprechend der jeweiligen okulären Vorerkrankung. Hinweise auf SARS-CoV-2 assoziierte entzündliche Prozesse waren nicht nachweis- 


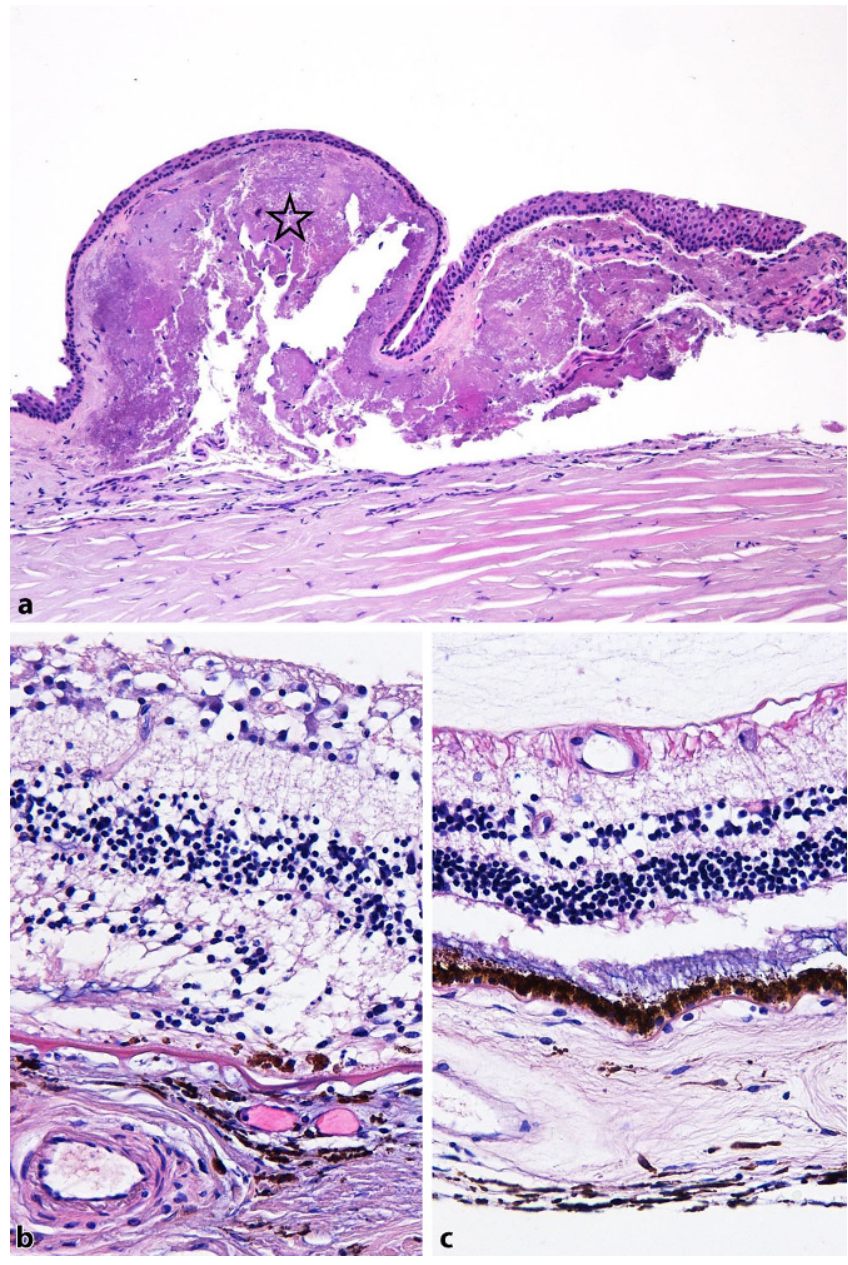

Abb. $3<$ (Patient 3, RA, Paraffinschnitt, Hämatoxylin-Eosin-Färbung, Vergr. 100:1). a Limbusnahe Bindehaut mit deutlicher Pinguecula (Stern), aber ohne Entzündung; b Netzhaut im Bereich der Makula mit fortgeschrittener Atrophie der Photorezeptoren, des retinalen Pigmentepithels und der Aderhaut im Sinne einer altersabhängigen Makuladegeneration; c Netzhaut in der Peripherie ohne entzündliche Veränderungen bar. Die äußerst milde konjunktivale Entzündungszellinfiltration unterschied sich nicht von einem ansonsten üblichen postmortalen Befund, zumal diese Patienten alle auf einer Intensivstation verstarben und es hier häufig zu einer unspezifischen Bindehautchemosis/ Injektion kommt.

\section{Diskussion}

Das neue Coronavirus SARS-CoV-2 stellt für uns alle eine große Herausforderung dar. Jeden Tag werden wir konfrontiert mit neuen Zahlen, die allerdings nicht unbedingt systematisch erhoben werden. Laut Robert Koch-Institut (RKI) liegt die Inkubationszeit im Mittel bei 5 bis 6 Tagen mit einer Spannweite von 1 bis 14 Tagen [2, 3]. Als Hauptübertragungswege gelten laut RKI Tröpfchen und Aerosole [4-6]. Neben typischen Grippesymptomen wie Fieber und Halsschmerzen kann die Erkrankung zu schweren Organbeteiligungen - insbesondere der Lunge - mit akutem respiratorischem Distress-Syndrom (ARDS) führen, oft verkompliziert durch superponierte Bronchopneumonien. COVID19-Patienten haben auch eine ausgeprägte Thrombose-/Mikrothrombose-/ Thrombembolieneigung [7-9]. Zu weiteren Organbeteiligungen ist wenig in der Literatur beschrieben, und eine Augenbeteiligung bei SARS-CoV-2 ist bislang nur als Konjunktivitis erwähnt. Je genauer wir das Virus und seine Interaktionen mit dem Menschen allerdings kennen, umso besser können wir uns hoffentlich - auch davor schützen oder sogar therapeutische Ansätze finden.

Man unterscheidet bei den Coronaviren verschiedene Subtypen, die sowohl verschiedene Tierspezies als auch den Menschen befallen - jeweils mit durchaus unterschiedlicher Manifestation [10]. Bislang sind 7 humanpathogene Coronaviren bekannt, wobei insbesondere SARS-CoV, MERS-CoV und SARS-CoV-2 eine lebensbedrohliche Erkrankung verursachen können [10]. Im Rahmen weiterer Symptome einer SARS-CoV-2-Infektion, aber auch der möglichen Ansteckungswege wurde insbesondere die Bindehaut bzw. eine Konjunktivitis diskutiert [11-16]. Von der Tatsache, dass ein anderes Coronavirus (SARS-CoV) am Auge nachgewiesen wurde [17], lässt sich nicht automatisch rückschließen, dass dies auch für das jetzt agierende Coronavirus gilt, auch wenn die Viren sich sehr ähnlich sind. Nach der vorliegenden Literatur lässt sich das Virus - wenn überhaupt - nur bei Patienten mit Konjunktivitis nachweisen [11], die aber wiederum kein klassisches Symptom der COVID-19Infektion darstellt.

Es gibt recht eindeutige Hinweise darauf, dass SARS-CoV-2 den ACE2-Rezeptor als Andockstelle zur Penetration der Zellmembran nutzt [18, 19]. Diese Rezeptoren wurden auch in der Bindehaut des menschlichen Auges bereits beschrieben [20,21], wenngleich auch in nichtsignifikantem Ausmaß [21]. Eine mögliche Beteiligung der Bindehaut im Rahmen der Infektionskette könnte man daher zwar theoretisch erwarten, dies scheint angesichts der vorliegenden Ergebnisse jedoch eher unwahrscheinlich [21].

Zusätzlich könnten andere Faktoren wie ein reduziertes Bindungspotenzial oder insbesondere auch ein protektiver Effekt der Umgebung (der Tränenfilm enthält viele Substanzen, die hier potenziell interagieren könnten) für das spezifische Viruspotenzial von SARS-CoV-2 hinsichtlich einer okulären Infektion eine entscheidende Rolle spielen.

Im klinischen Alltag gilt die akute Konjunktivitis als eine der häufigsten okulären Erkrankungen, und in den allermeisten Fällen beruht sie auf einer viralen Infektion [22, 23]. Dies macht es zusätzlich extrem unwahrscheinlich, einen entsprechenden COVID-19-Patienten allein an diesem Symptom identifizieren zu können. Die Adenoviren Typ 8, 19, 37, Erreger der hochansteckenden Keratoconjunctivitis epidemica, sind im Bindehautabstrich nachweisbar und können somit auch über das Tränensekret 
übertragen werden. Im Gegensatz dazu scheint eine konjunktivale Übertragung bei COVID-19-Patienten (bei denen der Erreger bislang nur in Einzelfällen im Bindehautabstrich nachweisbar war) nach bisheriger Datenlage eher unwahrscheinlich, auch wenn es nicht gänzlich auszuschließen ist. Aus unseren Postmortem-Befunden von 3 Augenpaaren können wir für die Bindehaut derzeit weder eine spezielle diagnostische noch eine infektiöse Rolle ableiten, insbesondere da uns auch keine entsprechenden molekulargenetischen Daten vorliegen. Möglicherweise war eine Konjunktivitis zum Todeszeitpunkt nahezu ausgeheilt und damit nicht mehr histologisch als solche $\mathrm{zu}$ diagnostizieren. Klinische Befunde und entsprechende Bindehautabstriche bei SARS-CoV-2-positiven Patienten sowie entsprechende Untersuchungen zur konjunktivalen Übertragung und Pathogenität des Erregers sollten zukünftig hier mehr Klarheit bringen. Erfreulicherweise fanden sich aber auch keine Hinweise auf eine direkte Mitbeteiligung anderer okulärer Strukturen im Rahmen von COVID-19.

Zusammenfassend lässt sich aus unseren Befunden keine generelle Empfehlung über die grundsätzlichen $\mathrm{Hy}$ gienemaßnahmen hinaus oder eindeutige Erkenntnisse bezüglich einer möglichen Augenbeteiligung gewinnen, und wir sind uns bewusst, dass wir mit unserem Beitrag an negativen Befunden nur einen sehr kleinen Baustein zu diesem komplexen Krankheitsbild liefern können. Nach den vorliegenden Daten - auch unter Einbeziehung der aktuellen Literatur - scheint das Auge zumindest in der akuten Situation (abgesehen von einer gelegentlich auftretenden Konjunktivitis) kein pathologisch relevantes Zielorgan zu sein.

\section{Zusammenfassung}

Bei den von uns untersuchten Augen von an COVID-19 verstorbenen Patienten zeigten sich keine eindeutigen Hinweise auf SARS-CoV-2-assoziierte entzündliche Prozesse. Die äußerst milde konjunktivale Entzündungszellinfiltration unterschied sich nicht von einem ansonsten üblichen postmortalen Befund.
Nach den vorliegenden Daten - auch unter Einbeziehung der aktuellen Literatur - scheint das Auge zumindest in der akuten Situation (abgesehen von einer gelegentlich auftretenden Konjunktivitis) kein pathologisch relevantes Zielorgan zu sein.

\section{Korrespondenzadresse}

Prof. Dr. med. Karin U. Löffler

Augenklinik, Universitätsklinikum Bonn

Ernst-Abbe-Str. 2, 53127 Bonn, Deutschland

Karin.Loeffler@ukbonn.de

Danksagung. Besonderen Dank gebührt Herrn Ralf Schoch aus dem Institut für Pathologie Basel für Unterstützung bei der Autopsie, insbesondere bei der Asservierung der Augen. Frau Claudine Strack danken wir für die Erstellung der histologischen Abbildungen.

\section{Einhaltung ethischer Richtlinien}

Interessenkonflikt. K.U.Löffler, A. Reinhold, M.C.Herwig-Carl, A. Tzankov, F.G. Holz, H.P.N. Scholl und P. Meyer geben an, dass kein Interessenkonflikt besteht.

Für diesen Beitrag wurden von den Autoren keine Studien an Menschen oder Tieren durchgeführt. Für die aufgeführten Studien gelten die jeweils dort angegebenen ethischen Richtlinien.

\section{Literatur}

1. Menter T, Haslbauer JD, Nienhold R et al (2020) Post-mortem examination of COVID19 patients reveals diffuse alveolar damage with severe capillary congestion and variegated findings of lungs and other organs suggesting vascular dysfunction. Histopathology. https://doi.org/10. $1111 /$ his.14134

2. Zhou B, She J, Wang Y et al (2020) Venous thrombosis and arteriosclerosis obliterans of lower extremities in a very severe patient with 2019 novel coronavirus disease: a case report. J Thromb Thrombolysis. https://doi.org/10.1007/s11239020-02084-w

3. WHO (Hrsg) (2020) Report of the WHO-China Joint Mission on Coronavirus Disease 2019 (COVID-19)

4. Leung NHL, Chu DKW, Shiu EYC et al (2020) Respiratory virus shedding in exhaled breath and efficacy of face masks. Nat Med. https://doi.org/10. 1038/s41591-020-0843-2

5. van Doremalen N, Bushmaker T, Morris DH et al (2020) Aerosol and Surface Stability of SARSCoV-2 as Compared with SARS-CoV-1. N Engl J Med 382(16):1564-1567. https://doi.org/10.1056/ NEJMc2004973

6. Wu Y-C, Chen C-S, Chan Y-J (2020) The outbreak of COVID-19: an overview. J Chin Med Assoc 83(3):217-220. https://doi.org/10.1097/JCMA. 0000000000000270

7. Giannis D, Ziogas IA, Gianni P (2020) Coagulation disorders in coronavirus infected patients: COVID 19, SARS-CoV-1, MERS-CoV and lessons from the past. J Clin Virol 127:104362. https://doi.org/10. 1016/j.jcv.2020.104362

8. Han H, Yang L, Liu Ret al (2020) Prominent changes in blood coagulation of patients with SARS-CoV-2 infection. Clin Chem Lab Med. https://doi.org/10. 1515/cclm-2020-0188

9. Tang N, Bai H, Chen X et al (2020) Anticoagulant treatment is associated with decreased mortality in severe coronavirus disease 2019 patients with coagulopathy. J Thromb Haemost 18(5):1094-1099. https://doi.org/10.1111/jth.14817

10. Seah I, Agrawal R (2020) Can the Coronavirus Disease 2019 (COVID-19) Affect the Eyes? A Review of Coronaviruses and Ocular Implications in Humans and Animals. Ocul Immunol Inflamm 28(3):391-395. https://doi.org/10.1080/ 09273948.2020.1738501

11. Xia J, Tong J, Liu M et al (2020) Evaluation of coronavirus in tears and conjunctival secretions of patients with SARS-CoV-2 infection. J Med Virol. https://doi.org/10.1002/jmv.25725

12. Liu Z, Sun C-B (2020) Conjunctiva is not a preferred gateway of entry for SARS-CoV-2 to infect respiratory tract. J Med Virol. https://doi.org/10. 1002/jmv.25859

13. Peng Y, Zhou Y-H (2020) Is novel coronavirus disease (COVID-19) transmitted through conjunctiva? JMed Virol.https://doi.org/10.1002/jmv.25753

14. Seah IYJ, Anderson DE, Kang AEZ et al (2020) Assessing viral shedding and Infectivity of tears in Coronavirus disease 2019 (COVID-19) patients. Ophthalmology. https://doi.org/10. 1016/j.ophtha.2020.03.026

15. Sun C-B, Wang Y-Y, Liu G-H et al (2020) Role of the eye in transmitting human Coronavirus: what we know and what we do not know. Front Public Health 8:155. https://doi.org/10.3389/ fpubh.2020.00155

16. Zhou Y, Duan C, Zeng Y et al (2020) Ocular findings and proportion with conjunctival SARS-COV-2 in COVID-19 patients. Ophthalmology. https://doi. org/10.1016/j.ophtha.2020.04.028

17. Loon S-C, Teoh SCB, Oon LLE et al (2004) The severe acute respiratory syndrome coronavirus in tears. $\mathrm{Br}$ J Ophthalmol 88(7):861-863. https://doi.org/10. $1136 /$ bjo.2003.035931

18. Stahlmann R, Lode H (2020) Medication for COVID19-an overview of approaches currently under study. Dtsch Arztebl Int 117(13):213-219. https:// doi.org/10.3238/arztebl.2020.0213

19. Hoffmann $M$, Kleine-Weber $H$, Schroeder $S$ et al (2020) SARS-coV-2 cell entry depends on ACE2 and TMPRSS2 and is blocked by a clinically proven protease. Inhib Cell 181(2):271-280.e8. https:// doi.org/10.1016/j.cell.2020.02.052

20. Zhang BN, Wang Q, Liu T et al (2020) Expression analysis of 2019-nCoV related ACE2 and TMPRSS2 in eye tissues. Zhonghua Yan Ke Za Zhi 56:E11. https://doi.org/10.3760/cma.j.cn11214220200310-00170

21. Lange C, Wolf J, Auw-Haedrich C et al (2020) Expression of the COVID-19 receptor ACE2 in the human conjunctiva. J Med Virol. https://doi.org/ 10.1002/jmv.25981

22. Sambursky RP, Fram N, Cohen EJ (2007) The prevalence of adenoviral conjunctivitis at the Wills Eye Hospital Emergency Room. Optometry 78(5):236-239. https://doi.org/10.1016/j.optm. 2006.11.012

23. Woodland RM, Darougar S, Thaker U et al (1992) Causes of conjunctivitis and keratoconjunctivitis in Karachi, Pakistan. Trans R Soc Trop Med Hyg 86(3):317-320. https://doi.org/10.1016/00359203(92)90328-a 